\title{
Phase Separation of a Binary Fluid in the Inertia-Dominated Regime
}

\author{
Anirban Sain* and Martin Grant \\ Physics Department, McGill University, Rutherford Building, 3600 rue University, Montreal, QC, Canada H3A 2T8
}

(Received 9 August 2004; published 16 December 2005)

\begin{abstract}
The phase separation kinetics of a binary fluid is studied analytically through an effective one-fluid model with a random force spectrum determined self-consistently: the rate of kinetic energy injection by the random force is consistent with the droplet coalescence rate. Our detailed results for the rates of energy dissipation and kinetic energy decay are consistent with previous numerical studies. We find that simple nontrivial scaling, if any, is associated with a new universality class where the velocity length scale follows $L_{v} \sim t^{1 / 2}$.
\end{abstract}

PACS numbers: 64.60.My, 05.70.Ln, 64.60.Cn, 81.30.Hd

The late-stage kinetics of phase separation in a binary fluid is poorly understood. This is in contrast to the understanding of phase separation in, for example, binary alloys, where dynamical scaling occurs characterized by a few universal attributes. Following preparation in an initially mixed state, the two phases separate, and domains of either phase form and grow with time $t$ via the characteristic length $\bar{R}(t) \sim t^{\alpha}$, for late times, where $\alpha$ is the growth exponent. For a conserved (nonconserved) scalar order parameter, $\alpha=1 / 3(1 / 2)$, corresponding to the universality classes of models $B$ and $A$, respectively. Universality classes for first-order phase transition kinetics are determined by, at least, the presence or absence of conservation laws for the ordering field, and are characterized by, e.g., the value of $\alpha$, and the scaled form of correlation functions. Whether or not there are additional universality class for fluids, due to the presence or absence of fluid convection, is unknown. For the related phenomena of dynamics near a continuous phase transition, fluid convection is indeed relevant, as it is due to a Poisson-brackets relation; the universality class is distinct and is called model $H$ [1] in this context. For phase separation in fluids, however, the existence of a late-stage scaling regime has not been definitively established, and, in particular, nor has the value of the growth exponent characterizing that regime. Distinct growth laws have been proposed for fluids, based upon dimensional and physical arguments. Of particular note is the effective behavior [2] $\bar{R}(t) \sim t$ at initial times in three dimensions when motion is dominated by fluid viscosity (and $\bar{R}(t) \sim t^{1 / 2}$ in two dimensions [3]), and the proposal for the asymptotic regime [4] $\bar{R}(t) \sim t^{2 / 3}$, when growth is dominated by fluid inertia. However, one of us and Elder [5] have argued, using dimensional and scaling arguments, that the asymptotic exponent must obey $\alpha \leq 1 / 2$.

Here, we address this issue through a novel analytic approach. We introduce an effective one-fluid description for the fluid velocity $\mathbf{v}$ driven by noise. The spectrum of that noise is determined self-consistently, describing, for example, the coalescence of droplets through the growth exponent $\alpha$. To investigate the possibility of scaling of the velocity field, we define a length scale $L_{v} \sim t^{1 / z}$, where simple nontrivial scaling evidently corresponds to $\alpha=$ $1 / z$. The solution of the linearized equation gives qualitative and quantitative agreement with the results of simulations [6,7]. The relevance of nonlinearities is examined though dimensional analysis, a Ginzburg criterion, and a constraint imposed by Galilean invariance. We find that either there is no simple nontrivial scaling between droplet growth and the velocity field (that is, the droplets trivially follow the universality class of model $B$ with $\alpha=1 / 3$, while the velocity field obeys $1 / z=1 / 2$ ), or scaling is obeyed with the previously obtained bound corresponding to the new universality class of model $H$, which we argue would be satisfied as an equality $\alpha=1 / z=1 / 2$.

Simulations have focused mostly upon the order parameter field $\psi(\mathbf{x}, t)$, as a function of spatial position $\mathbf{x}$ and time. Although still not in a position to reach the asymptotic regime, simulations have been able to verify the existence of transient regimes $\alpha \approx 1$, and $\alpha \approx 2 / 3$. Recently, a study of the velocity field $\mathbf{v}(\mathbf{x}, \mathbf{t})$ has been reported in a large three-dimensional lattice Boltzmann simulation by Kendon et al. [6,7], obtaining the velocity spectrum $S_{v}(k, t) \equiv\left\langle|\hat{\mathbf{v}}(k, t)|^{2}\right\rangle$, where $\hat{\mathbf{v}}(k, t)$ is the Fourier transform of $\mathbf{v}(\mathbf{x}, t)$, with respect to the wave number $\mathbf{k}$. From this, one obtains $L_{v}(t)$. Kendon et al. showed that the order parameter structure function $S_{\psi}(k, t) \equiv\left\langle|\hat{\psi}(\mathbf{k}, t)|^{2}\right\rangle$ retains its scaling form as it passes from viscous to inertial regimes. In contrast, $S_{v}(k, t)$ changed substantially. They reported that $1 / z$ remains $2 / 3$ even though $\alpha$ changes from 1 to $2 / 3$. Although they did not find evidence for a $1 / z=$ $1 / 2$ regime, they did not rule out the possibility of such an asymptotic behavior. This indicates that the order parameter field behaves independently of the velocity field, while the velocity depends upon the order parameter.

Hence, we study the evolution of the velocity field driven by the $\psi(x)$ field. Our picture is simple: fluid near droplet interfaces is moved as droplets coalesce, so that the droplet growth injects kinetic energy into the fluid. For example, when two droplets coalesce, the "neck" connecting them disappears as the fluid surrounding them is pushed away: the total interfacial area is reduced, and converted to fluid kinetic energy. We model this behavior 
of the fluid by the Navier-Stokes equation in the incompressible limit

$$
\dot{\mathbf{v}}+\lambda(\mathbf{v} \cdot \nabla) \mathbf{v}=\nu \nabla^{2} \mathbf{v}(\mathbf{x})+\mathbf{f},
$$

where $\lambda$ and $\nu$ are constants, and the external random force $\mathbf{f}$ is due to the fluctuations in $\mathbf{v}$ induced by droplet coalescence. Other motivations, such as an explicit coarse graining of the order parameter, should also lead to this form, as the nonlinear Navier-Stokes equation arises from general considerations. What remains is to determine the form of $\mathbf{f}$ due to the fluctuations induced by the order parameter, that is, due to the droplets. While each particular coalescence of droplets generates a fixed pattern, given the radii and local velocity of each droplet, a superposition of the motion of many droplets creates a random flow pattern $[8,9]$. As well, it is natural for the random force to be peaked around scale $\bar{R}(t)$ : the random velocity pattern (and ensuing fluctuation) generated by each coalescence event is determined by the smaller of the two participating droplets [8], which are both of size proportional to $\bar{R}(t)$. This gives rise to a scaled noise intensity evolving as the droplets grow. Hence we model $\mathbf{f}$ as a Gaussian random force in space and time, whose second moment obeys:

$$
\left\langle\hat{f}_{i}(\mathbf{k}, t) \hat{f}_{j}\left(\mathbf{k}^{\prime}, t^{\prime}\right)\right\rangle=P_{i j} D(k, t) \delta\left(\mathbf{k}+\mathbf{k}^{\prime}\right) \delta\left(t-t^{\prime}\right),
$$

where the transverse projection operator $P_{i j}(\mathbf{k})=\delta_{i j}-$ $k_{i} k_{j} / k^{2}$ enforces incompressibility, and the amplitude of the noise $D(k, t)$ is to be determined. Such a form implies the neglect of short-length-scale, short-time-scale correlations between coalescence events; this is permissible since such correlations are transient, and we are investigating asymptotic behavior. The noise amplitude $D$ arises from the droplet coalescence events which conserve momentum, so $D \propto k^{2}$ as $k \rightarrow 0$. We will look for solutions which are scale invariant. As mentioned above, in related contexts and at least to good numerical approximation in the present context [7], the order parameter correlations satisfy dynamical scaling, with power laws for large and small $k$. Hence we will look for a self-consistent scaling solution with

$$
D=D_{0} \bar{R}^{\beta} \frac{(k \bar{R})^{2}}{\left[1+(k \bar{R})^{2}\right]^{n}},
$$

where $D_{0}$ is a constant, and $\bar{R} \propto t^{\alpha}$, so that the spectrum of the velocity fluctuations is driven by a prescribed spectrum of droplet coalescence. The exponents $n=\frac{1}{2}(d+1)+1$, in $d$ dimensions, and $\beta=d-1-\frac{1}{\alpha}$, as described below. The prefactor $D_{0}$ is a function of the constant volume fraction, such that $D_{0}$ vanishes as the volume fraction approaches 0 or 1 , since the driving force for demixing vanishes in those limits.

We obtain the values of $n$ and $\beta$ by considering the rate of energy injection at scale $k: I_{k} \propto k^{d-1}\langle\mathbf{v}(k) f(-k)\rangle$. $I_{k}$ can be determined using an algebraic identity $[10]\langle v(t) f(t)\rangle=$ $\Omega / 2$, valid for a Langevin equation of the form $\dot{v}=$
$F(v)+f$, with Gaussian noise correlation $\left\langle f(t) f\left(t^{\prime}\right)\right\rangle=$ $\Omega \delta\left(t-t^{\prime}\right)$. The total rate of energy injection is thus $\int d^{d} k D(k, t)$. Using isotropy, the integrand follows $k^{d+1-2 n}$ for large $k$. Hence, we must have $n=\frac{1}{2}(d+1)+$ $m$, where $m \geq 1$, and we choose $m=1$. Our main results are not sensitive to this choice [11]. This ensures that $I_{k}$ does not diverge. In this context, we recall that in the coarse-grained model for a stirred fluid near equilibrium [12], the force variance is assumed to be proportional to $k^{2}$ such that equipartition is reached; in that case the force is cut off at a microscopic length scale beyond which the continuum description using the Navier-Stokes equation fails. To determine the value of $\beta$, we invoke physical arguments. Consider the case of phase separation where the minority phase consists of spherical droplets. Then the total volume of that phase is $\int_{0}^{\infty} R^{d} N(R, t) d R=$ const, where $N(R, t)$ is the number of droplets of size $R$ per unit volume. Scaling and volume conservation implies $N(R, t) \propto N^{*}(R / \bar{R}) / \bar{R}^{d+1}$, where $N^{*}(x)$ is a universal function [13]. This allows us to predict the net rate of energy conversion from interfacial free energy to the kinetic energy of fluid - the kinetic energy injection rate $I_{T}$. This is given by the negative of the rate of change of total interfacial energy. The total interfacial energy stored in all the droplets is $E_{T}=\int \bar{R}^{d-1} N(R, t) d R \propto t^{-\alpha}$, Thus, $I_{T}=$ $-\dot{E}_{T}(t) \propto t^{-(1+\alpha)}$. Scaling implies this is the form of the rate of loss of surface area, without regard to the consideration of spherical droplets. This energy feeds into the Navier-Stokes equation giving the total kinetic energy injection rate due to the Gaussian noise, which is $\int I_{k} d^{d} k \propto$ $\bar{R}^{\beta-d} \propto t^{\alpha(\beta-d)}$. Matching the time dependence of these two rates we obtain $\beta=d-1-\frac{1}{\alpha}$.

First we neglect the convective nonlinearity, and consider $d=3$. Although we will find directly below that the nonlinearities are relevant, the linear case gives good qualitative insight into the phenomena, and, at least, describes crossover transients. In terms of the scaled variable $u=k^{1 / \alpha} t$ we obtain

$$
S_{v}(k, t)=\left(\frac{t}{u}\right)^{2 \alpha} \int_{0}^{u} d u^{\prime} \frac{u^{\prime 4 \alpha-1} e^{-2 \eta\left(u-u^{\prime}\right)(u / t)^{2 \alpha-1}}}{\left[1+u^{\prime 2 \alpha}\right]^{3}},
$$

in the linear regime, from which we obtain $L_{v}(t) \equiv$ $\int S_{v}(k, t) d^{3} k / \int k S_{v}(k, t) d^{3} k \sim t^{1 / z}$, with the latter asymptotic result corresponding to late times. The choice $\alpha=$ $1 / 2$ gives scaling, where $S_{v}$ can be expressed in terms of exponential integral functions, with $S v(k, t) / t^{2 \alpha}$ being a universal function of only $u=k^{1 / \alpha} t$. Substituting the scaling form of $S_{v}$ with $\alpha=1 / 2$, we obtain $L_{v} \sim t^{1 / 2}$. Choosing $\alpha=1,2 / 3$, or any $\alpha>1 / 2$, one obtains $L_{v}(t) \sim t^{1 / 2}$, for sufficiently large $t$. Consider $S_{v}$. Scaling requires that $S_{v}(k, t) / t^{2 \alpha}$ be a universal function of only $u=k^{1 / \alpha} t$. For the linear case, for $\alpha=1 / 2$, this is satisfied, but not for $\alpha=1$ or $2 / 3$. See Fig. 1. Indeed, for $u \gg$ 1 , irrespective of $\alpha$, the integral obtains its main contribu- 


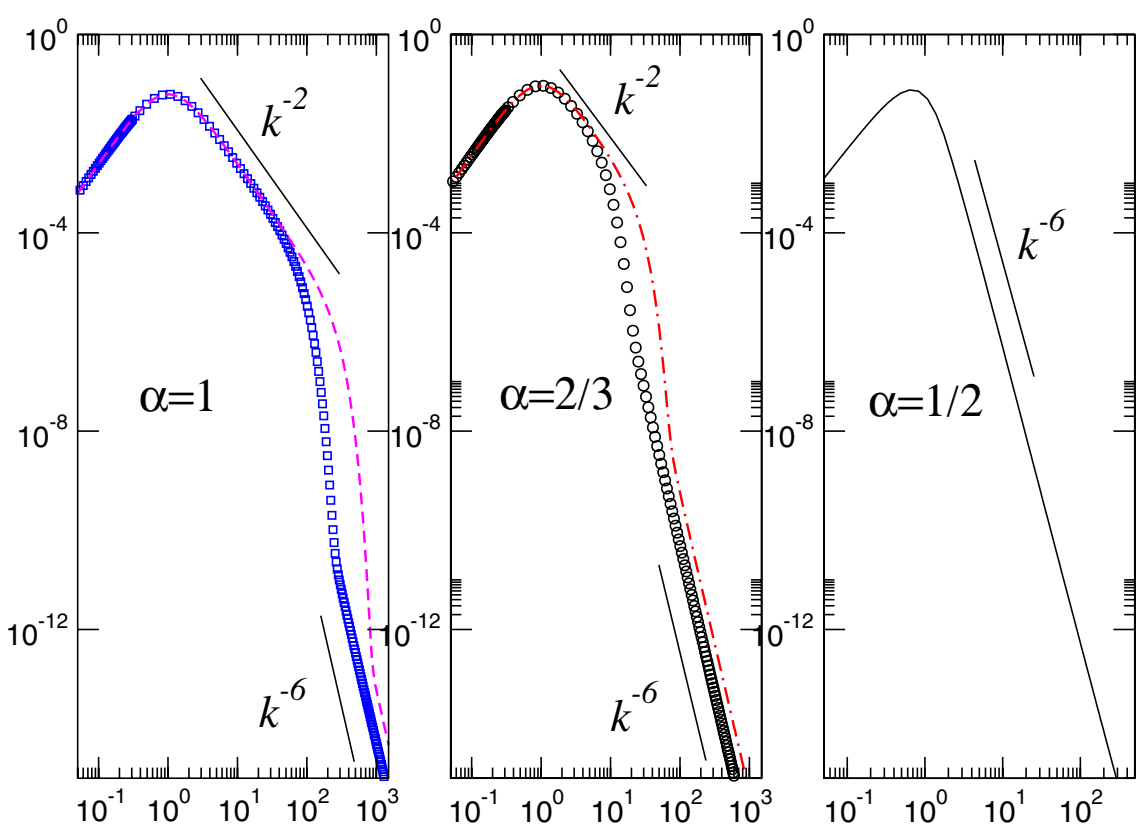

FIG. 1 (color online). Linear result for $S_{v}(k, t) / t^{2 \alpha}$ versus $k t^{\alpha}\left(=u^{\alpha}\right)$, for different $\alpha$ 's and $t$ 's. $\alpha=1: t=10^{5}$ (dashed line), $t=10^{4}$ (square); $\alpha=2 / 3: t=10^{9}$ (dash-dotted line), $t=10^{6}$ (circle); and $\alpha=1 / 2$, independent of $t$ (solid line). Note that only the structure function for $\alpha=1 / 2$ exhibits universal behavior.

tion from $u^{\prime}=u$, and scales as $k^{-6}$, as shown in three dimensions. There is a $k^{-2}$ contribution to the spectrum for $\alpha>1 / 2$, which exists for large $u>1$ bounded by $u<$ $\mathcal{O}\left(t^{(2 \alpha-1) / 2 \alpha}\right)$. This regime is bounded at small $k$ by a $k^{2}$ spectrum, and at large $k$ by $k^{-6}$ tails. We note that the $k^{-2}$ scaling evident in the viscous regime $(\alpha=1)$ was reported by Kendon et al. [7]. Figure 1 also shows that $S_{v}$, for $\alpha=$ $2 / 3$, shows a growing $k^{-2}$ regime as time evolves; a similar trend can be observed in Fig. 4(b) of Ref. [7].

One can further consider the linear case as follows. From the equation of motion one can show that the kinetic energy satisfies $E_{\mathrm{KE}}(t) \sim t^{-1 / 2}$ for large $t$ for $\alpha \geq 1 / 2$. As well, for all $\alpha$, one can show that the kinetic energy of the fluid decays slowly, while $L_{v}$ grows. The root-mean-square velocity $v_{\mathrm{rms}}=\sqrt{E_{\mathrm{KE}}}$ always decreases, implying that the Taylor microscale Reynolds' number [10] $\mathrm{Re}_{T} \sim$ $v_{\mathrm{rms}}^{2} /\left[\eta \sqrt{\left\langle\left(\nabla_{i} v_{j}\right)\left(\nabla_{i} v_{j}\right)\right\rangle}\right]$, does not saturate and still increases, following $t^{1 / 4}$ for $\alpha=1 / 2$, increasing faster for $\alpha=1$ and 2/3. This is indeed what is seen for $\operatorname{Re}_{T}$ in the simulations of Kendon et al. [7]. They also defined two other Reynolds numbers, neither of which saturated for $\alpha=2 / 3$. Their extended scaling theory predicted that one of these numbers should saturate in the true asymptotic regime, leading them to conclude that the true asymptotic regime was not yet been reached in their simulations. Indeed, this is consistent with our results, as our linear theory (which gives qualitative and quantitative agreement with their results) describes only transients.

The relevance of nonlinearities can be shown from dimensional analysis of quantities with respect to length
$L$, as is standard in the study of critical phenomena [1]. We focus on the dependence of quantities on the characteristic length $L$, and so scaling does not involve the bare coupling constants $\nu, \lambda$, or $D_{0}$, and, in particular, on the asymptotic large- $L$ behavior. A Ginzburg criterion determines the relevance or irrelevance of nonlinear convection on those scales. Explicitly, we define dimensions of characteristic quantities as $[t]=L^{z}$, and $[v]=L^{\chi^{-1}}$, such that the Reynolds' number [14] is $\operatorname{Re}=L^{\chi}$. Therefore, the sign of $\chi$ determines the Ginzburg criterion: if $\chi<0$, convection is asymptotically irrelevant for large $L$, if $\chi>0$, convection is relevant. The critical dimension $d_{c}$ is the dimension where $\chi$ changes sign. This is important to identify for the potential application of the renormalization group as one can, for example, perturbatively investigate nonlinear behavior in an expansion in $d-d_{c}=\epsilon$.

In the linear regime, where we neglect the convective nonlinearity, we immediately have $z=2$, of course. Hence, neglecting the convective term, there is no nontrivial scaling of the velocity field with the droplet growth unless $\alpha=1 / 2$, as was evident above, in, for example, Fig. 1. The relevance of the nonlinear term can be obtained from the Reynolds number $\operatorname{Re}=L^{\chi}$ as $L \rightarrow \infty$. As the Ginzburg criterion, we obtain, for $\alpha<\frac{1}{2}, \chi=-\left(\frac{1}{2}-\alpha\right) \times$ $\left(d-d_{c}\right)$ with the critical dimension $d_{c}=2 \alpha /(1-2 \alpha)$; for $\alpha=\frac{1}{2}, \quad \chi=\frac{1}{2}$; and for $\alpha>\frac{1}{2}, \quad \chi=\frac{3}{2}-2 \alpha$ using $m=1$.

It is worthwhile to discuss the physical implications of these three cases. For $\alpha<1 / z$ the droplets grow on a much slower time scale than the fluid's motion. Hence we expect droplet growth to be trivially identical to the overdamped 
case, i.e., identical to the well-known case of the conserved scalar order parameter where $\alpha=1 / 3$. This value of $\alpha=$ $1 / 3$ implies a critical dimension of $d_{c}=2$, so we expect $[15,16]$ the linear theory to hold for the velocity field in $d=3$, with possible logarithmic corrections in $d=2$. It is interesting to note that $d_{c} \rightarrow \infty$ as $\alpha \rightarrow(1 / z)^{-}$.

The case $\alpha>1 / z$ is unphysical for fluid unmixing, as the droplet interfaces move faster than the velocity field. Nevertheless, we should note that a nonscaling result for fluid unmixing might indeed involve several lengths where this limit might play a role. In this regard it is interesting, though irrelevant in the present context, to note that $\chi$ becomes negative and stabilizing for sufficiently large $\alpha \geq \frac{3}{4}$, for $m=1$.

Simple nontrivial scaling of the droplets with the velocity field implies $\alpha=1 / z$. Linear theory gives $\operatorname{Re}=L^{1 / 2}$ for the Ginzburg criterion, so the convective nonlinearity is relevant in all dimensions, and is not perturbable by an $\epsilon=$ $d-d_{c}$ expansion. In this case, the linear results describe transients.

To further investigate this latter potential nonlinear scaling regime with $\alpha=1 / z$, we make use of an invariance of the equation of motion. As is the case for the noisy Burgers' equation and the equivalent Kardar-ParisiZhang equation [15], the equation of motion is invariant under a Galilean transformation involving a constant velocity $\mathbf{v}_{0}: \mathbf{v} \rightarrow \mathbf{v}-\mathbf{v}_{0}$, and $\mathbf{x} \rightarrow \mathbf{x}+\mathbf{v}_{0} t$. This implies the dimension of the coefficient $\lambda$ is constant, and so $\chi+z=$ 2 . Hence for the Reynolds number in the nonlinear regime: $\operatorname{Re}=L^{\chi}=L^{2-z}$. Using $\alpha=1 / z$ gives

$$
\operatorname{Re}=t^{2 \alpha-1},
$$

as obtained from a different approach in previous work [5]. But the system is equilibrating, so Re cannot increase with time or length scale. This implies $\chi \leq 0$, and $\alpha \leq 1 / 2$. In view of the linear results, e.g., $d_{c} \rightarrow \infty$ as $\alpha \rightarrow(1 / z)^{-}$, we expect these inequalities to be satisfied at their bounds, that is, $\alpha=1 / 2$, with $\chi=0$, if this nontrivial scaling regime exists.

In summary, we have presented a new approach for studying the phase separation of fluids, where we map a two-fluid description onto a nonlinear one-fluid model. The noise spectrum of that model is determined by enforcing its consistency with that of droplet coalescence. We obtained analytic results for the linear and nonlinear regimes. If droplet growth and the fluid velocity do not follow the same time dependence, we find that the fluid velocity is irrelevant to unmixing: the droplet scaling behavior is trivially the same as the overdamped case with $\alpha=1 / 3$, corresponding to the universality class of model $B$, and $z=$ 2 , the latter as described by linear theory, for dimensions $d \geq 2$. We find that simple nontrivial scaling of the droplet growth and the fluid motion, occurs only for $\alpha=1 / z \leq$ $1 / 2$, which we argue is satisfied at the equality, in a non- linear regime corresponding to the new universality class of model $H$.

One of us (A. S.) thanks M.E. Cates and J. Yeomans for discussion and Rahul Pandit for pointing out the simulation work by M.E. Cate's group. This work was supported by the Natural Sciences and Engineering Research Council of Canada, and le Fonds Québécois de la recherche sur la nature et les technologies.

*Present address: Physics Department, IIT Bombay, Powai, 400076, Mumbai, India.

Electronic address: asain@ phy.iitb.ac.in

[1] P. C. Hohenberg and B. I. Halperin, Rev. Mod. Phys. 49, 435 (1977).

[2] E. Siggia, Phys. Rev. A 20, 595 (1979).

[3] M. San Miguel, M. Grant, and J. D. Gunton, Phys. Rev. A 31, 1001 (1985).

[4] H. Furukawa, Phys. Rev. A 31, 1103 (1985).

[5] M. Grant and K. R. Elder, Phys. Rev. Lett. 82, 14 (1999).

[6] V. M. Kendon, J.C. Desplat, P. Blandon, and M. Cates, Phys. Rev. Lett. 83, 576 (1999).

[7] V. M. Kendon, M. Cates, I. Pagonabarraga, J.C. Desplat, and P. Blandon, J. Fluid Mech. 440, 147 (2001).

[8] J. Eggers, J. Lister, and H. A. Stone, J. Fluid Mech. 401, 293 (1999).

[9] Note that this approach is for dimensions larger than 1 , as there is no phase separation at finite temperature for $d \leq 1$.

[10] J. Zinn-Justin, Quantum Field Theory and Critical Phenomena (Clarendon, Oxford, 1989), p. 59; This relation is also known as Novikov's theorem in U. Frisch, Turbulence: The Legacy of A. N. Kolmogorov (Cambridge University Press, Cambridge, England, 1995).

[11] For completeness, we have also considered the case of variable $m$, as well as the possibility of exponential decay of the noise amplitude with wave number. These do not affect our results for the physical regime of the phenomena under study, that is for $\alpha \leq 1 / z$, where the velocity of the droplets is no larger than that of the surrounding fluid. Nevertheless, there may be interest in such a limit of the model in other contexts.

[12] D. Forster, D. R. Nelson, and M. J. Stephen, Phys. Rev. A 16, 732 (1977).

[13] J. H. Yao, K. R. Elder, H. Guo, and M. Grant, Phys. Rev. B 47, 14110 (1993).

[14] We define the Reynolds number as the dimensional dependence of the ratio of the convective to the dissipative term in the Navier-Stokes equation: $\operatorname{Re} \equiv\left[v \nabla v / \nabla^{2} v\right]=$ $[L v]=L^{\chi}$. Note that we focus on the dependence of these quantities on the characteristic length $L$, and so this scaling does not involve the bare coupling constants $\nu$ or $\lambda$.

[15] M. Kardar, G. Parisi, and Y.-C. Zhang, Phys. Rev. Lett. 56, 889 (1986).

[16] We note that $d_{c}$ may correspond to a lower critical dimension involving a phase transition from a linear regime at low $\lambda$, to a nonlinear regime obeying Galilean invariance at large $\lambda$. 